\title{
Systematic review of pressurized intraperitoneal aerosol chemotherapy for the treatment of advanced peritoneal carcinomatosis
}

\author{
F. Grass ${ }^{1}$, A. Vuagniaux ${ }^{1}$, H. Teixeira-Farinha ${ }^{1}$, K. Lehmann ${ }^{2}$, N. Demartines ${ }^{1}$ and M. Hübner $^{1}$ \\ Departments of Visceral Surgery, ${ }^{1}$ University Hospital of Lausanne (CHUV), Lausanne, and ${ }^{2}$ University Hospital Zurich, Zurich, Switzerland \\ Correspondence to: Pr. N. Demartines, Department of Visceral Surgery, CHUV, 46 Rue du Bugnon, CH-1011 Lausanne, Switzerland \\ (e-mail: demartines@chuv.ch)
}

\begin{abstract}
Background: Pressurized intraperitoneal aerosol chemotherapy (PIPAC) is a minimally invasive approach under investigation as a novel treatment for patients with peritoneal carcinomatosis of various origins. The aim was to review the available evidence on mechanisms, clinical effects and risks.

Methods: This was a systematic review of the literature on pressurized intraperitoneal chemotherapy published between January 2000 and October 2016. All types of scientific report were included.

Results: Twenty-nine relevant papers were identified; 16 were preclinical studies and 13 were clinical reports. The overall quality of the clinical studies was modest; five studies were prospective and there was no randomized trial. Preclinical data suggested better distribution and higher tissue concentrations of chemotherapy agents in PIPAC compared with conventional intraperitoneal chemotherapy by lavage. Regarding technical feasibility, laparoscopic access and repeatability rates were 83-100 and 38-82 per cent. Surgery-related complications occurred in up to 12 per cent. Postoperative morbidity was low (Common Terminology Criteria for Adverse Events grade 3-5 events reported in 0-37 per cent), and hospital stay was about 3 days. No negative impact on quality of life was reported. Histological response rates for therapy-resistant carcinomatosis of ovarian, colorectal and gastric origin were 62-88, 71-86 and 70-100 per cent respectively.

Conclusion: PIPAC is feasible, safe and well tolerated. Preliminary good response rates call for prospective analysis of oncological efficacy.

Paper accepted 1 February 2017

Published online in Wiley Online Library (www.bjs.co.uk). DOI: 10.1002/bjs.10521
\end{abstract}

\section{Introduction}

Peritoneal carcinomatosis remains a diagnostic and therapeutic challenge, and the prognosis for most underlying pathologies is dismal ${ }^{1-3}$. Although systemic chemotherapy has allowed tremendous improvements in the treatment of systemic metastases (especially liver and lungs), it appears to be much less effective for peritoneal spread mainly owing to pharmacokinetic limitations ${ }^{4}$, probably because of poor peritoneal vascularization impeding proper drug distribution ${ }^{5}$. Cytoreductive surgery (CRS) in combination with hyperthermic intraperitoneal chemotherapy (HIPEC) seems promising in highly selected patients ${ }^{6}$.

Pressurized intraperitoneal aerosol chemotherapy (PIPAC) has been proposed as a novel minimally invasive treatment for patients with advanced peritoneal carcinomatosis. PIPAC combines the benefits of a minimally invasive approach (easier repeat application, lower morbidity, better quality of life (QoL)) with the pharmacokinetic advantages of intraperitoneal administration (higher intratumoral concentrations, less systemic toxicity) and pressurized vaporization (better distribution and penetration $)^{7-10}$. The aim of the present study was systematically to review the scientific evidence on PIPAC with regard to mechanism, pharmacokinetics, safety and clinical effects.

\section{Methods}

\section{Data sources and search strategies}

Main electronic databases including MEDLINE (searched through PubMed), Embase, the Cochrane Database of Systematic Reviews and the Cochrane Central Register of Controlled Trials were searched. The medical 
subject heading (MeSH) terms used for searching PubMed were '(Intraperitoneal AND chemotherapy AND pressurized)'.

Further articles were sought by hand-searching electronic links to related articles and cross-referencing of selected articles. The trial registries http://clinicaltrials .gov, http://clinicaltrial.org, http://isrctn.org, http://actr .org.au, http://trialregister.nl and http://www.umin.ac .jp/ctr were screened for relevant unpublished prospective trials. The first description of a device to create intraperitoneal capnoperitoneum was published in $2000^{11}$. Therefore, the search was limited to studies published between January 2000 and October 2016.

\section{Study selection (inclusion and exclusion criteria)}

All types of original scientific report were considered. Reviews and book chapters were excluded. No language restrictions were applied. Only studies on pressurized intraperitoneal chemotherapy were included. Excluded from the analysis were reports on intraperitoneal chemotherapy by conventional lavage (HIPEC) or by use of an intraperitoneal catheter. The included articles were divided into preclinical and clinical studies.

\section{Data extraction and quality assessment}

Pertinent study characteristics (prospective versus retrospective, sample size, control group) were defined a priori and each article was assessed for potential sources of bias. Three authors independently performed the literature search. The search terms were identified first in the title, and then in the abstract or MeSH. All studies of interest were obtained as full-text articles and scrutinized thoroughly. The entire research team made the final decision on inclusion of each study. Relevant data were extracted and documented in a database developed $a$ priori. The following items were recorded: authors, title, year of publication, primary cancer, number of patients, number of PIPAC procedures, combination with other therapies, previous treatments, and surgical data (rate of non-access, surgery-related complications). Postoperative outcome measures were toxicity (assessed using the Common Terminology Criteria for Adverse Events, CTCAE) $)^{12}$, complications, mortality and follow-up. Tumour response was evaluated when possible by means of the objective tumour response (assessed using the revised Response Evaluation Criteria in Solid Tumours, RECIST) ${ }^{13}$, histological response, and overall and disease-specific survival. Tumour response (efficacy) was assessed consistently in patients who received at least one cycle of the study medication. Data are presented in accordance with the PRISMA statement ${ }^{14}$.

\section{Data analysis}

Statistical meta-analysis was not feasible as the original data were limited and heterogeneous. Therefore, descriptive statistics were applied and the available information was summarized in table form to provide a comprehensive overview of the most relevant aspects of mechanisms and clinical effects.

\section{Results}

The electronic search of the literature yielded 34 potential studies. By cross-referencing, 12 further studies were found. Of these 46 studies, five were excluded based on the title or abstract. Forty-one studies were evaluated as full-text articles. Of these, 12 were subsequently excluded owing to patient overlap or lack of new information: eight reviews $^{15-22}$, a textbook ${ }^{23}$ and a book chapter ${ }^{24}$. Two further studies were excluded owing to double publication: one ${ }^{25}$ reporting on safety aspects in German, which duplicated information in a paper from the same group written in English ${ }^{26}$, and one retrospective analysi ${ }^{27}$ of the whole Herne cohort with patient overlap with other publications from the same group (Fig. 1). MEDLINE search (PubMed) revealed most included articles; other data sources provided additional studies that were not indexed in $\mathrm{PubMed}^{28,29}$. Twenty-nine studies were retained for final analysis, 16 preclinical studies and 13 clinical reports (Tables 1 and $2)^{7-11,26-49}$. Of note, 23 of the 29 studies were performed by one working group from the pioneer centre in Herne, Germany; the remaining reports came from five other institutions.

Of the preclinical studies, 11 were animal studies, using in vivo (7) or ex vivo (4) models; all except two ${ }^{30,31}$ were porcine models. Two experimental studies ${ }^{33,38}$ dealt with human tissue samples and one study ${ }^{40}$ performed ex vivo granulometric analysis. One publication ${ }^{29}$ presented novel guidelines for histological tumour regression grading, and another study ${ }^{26}$ evaluated health aspects.

Clinical publications included 801 PIPAC procedures and 12 different primary tumours in 346 patients overall (Table 2). Five ${ }^{10,28,42,44,45}$ were prospective studies, including one phase II trial ${ }^{45}$, whereas $\operatorname{six}^{8,9,27,46,48,49}$ were conducted retrospectively. There were also two case reports ${ }^{43,47}$. No RCT has been conducted so far. Evidence for gynaecological, gastric and colorectal cancers is summarized in Table 3, along with limitations and potential biases owing to combined treatments. 


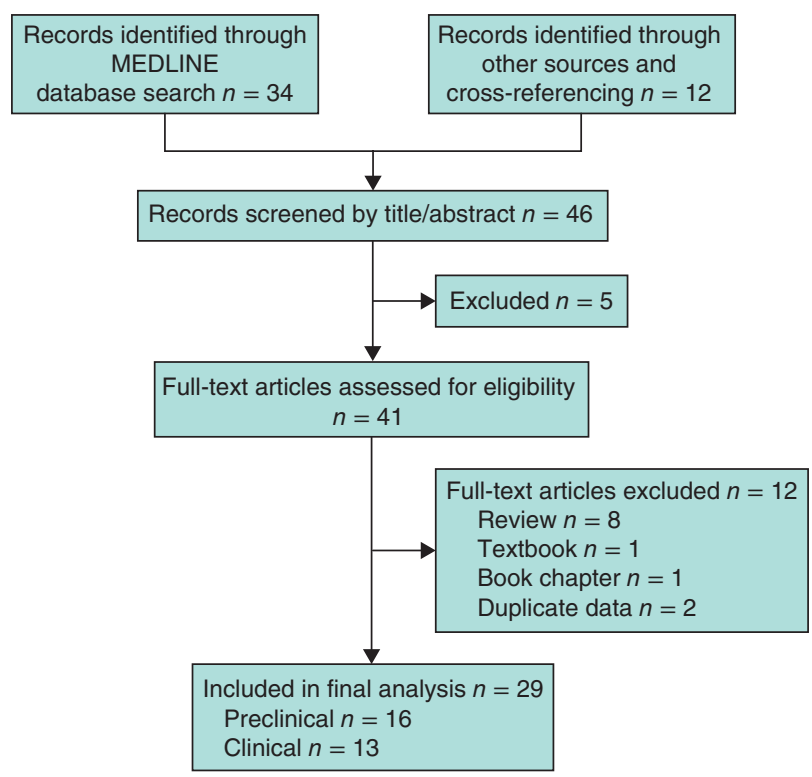

Fig. 1 PRISMA flow chart showing selection of articles for review

\section{Preclinical data}

\section{Development of the concept}

Three studies ${ }^{30-32}$ described the concept of intraperitoneal application of cytostatic solutions in gaseous form under pressure. A device to create therapeutic capnoperitoneum was first described in $2000^{11}$, but technical limitations prevented clinical application. A novel device to overcome these technical issues was presented a decade later ${ }^{7}$. Biodistribution of molecules applied by PIPAC and conventional lavage (HIPEC model) was then tested in an $e x$ vivo study ${ }^{33}$. In the aerosolized specimen, the test substance was found to be biologically active up to $1 \mathrm{~mm}$ depth, and showed more activity in tumour nodules than in normal peritoneum, whereas no activity was detected in the lavage samples ${ }^{33}$.

Conflicting results were reported by four recent studies ${ }^{37,39-41}$ on spatial drug distribution and tissue concentrations of PIPAC. First, doxorubicin was aerosolized within a hermetic container system with tissue samples at different locations ${ }^{37}$. Doxorubicin concentrations depended on location and were significantly higher at sites exposed directly to the aerosol jet of the nebulizer than at more distant $\operatorname{sites}^{41}$. Granulometric analysis under similar non-anatomical ex vivo conditions suggested a high percentage of droplets larger than $3 \mu \mathrm{m}$ favouring gravitational deposition close to the nebulizer, instead of homogeneous drug distribution ${ }^{40}$. Another similar experiment in the peritoneal cavity of a post-mortem swine simulated clinical conditions more precisely and reported more general dispersion of the drug, with penetration depths between $350 \mu \mathrm{m}$ in the centre and up to $150 \mu \mathrm{m}$ in more distant sites $^{39}$.

\section{Technique}

Surgical technique was standardized by the pioneer group $^{10,33}$ and conditions for PIPAC were defined empirically. Two balloon trocars (5 and $12 \mathrm{~mm}$ ) were inserted using an open technique or Veress needle to create a $12 \mathrm{mmHg}$ carbon dioxide capnoperitoneum. Alternatively, an Italian group ${ }^{8}$ described a single-port platform instead. Diagnostic laparoscopy included documentation of the extent of peritoneal carcinomatosis ${ }^{50,51}$. After removing the ascites, punch biopsies and peritonectomy specimens were retrieved for pathological examination. The nebulizer was then inserted via the $10-\mathrm{mm}$ trocar into the tight abdomen (as documented by zero-flow carbon dioxide) and connected to the pressure injector charged with liquid cytostatic solution. Oxaliplatin at a dose of $92 \mathrm{mg} / \mathrm{m}^{2}$ of body surface in $150 \mathrm{ml}$ dextrose solution was proposed for peritoneal carcinomatosis of colorectal origin. For peritoneal carcinomatosis of non-colorectal origin, a combination of cisplatin $\left(7.5 \mathrm{mg} / \mathrm{m}^{2}\right.$ in $150 \mathrm{ml} 0.9$ per cent sodium chloride) and doxorubicin $\left(1.5 \mathrm{mg} / \mathrm{m}^{2}\right.$ in $50 \mathrm{ml} 0.9$ per cent sodium chloride) was used. Conditions of nebulization were: application of cytostatics at a flow rate of $30 \mathrm{ml} / \mathrm{min}$, a pressure of $1380 \mathrm{kPa}$ over $30 \mathrm{~min}$, at room temperature $\left(22^{\circ} \mathrm{C}\right)$. After $30 \mathrm{~min}$, the capnoperitoneum was evacuated by use of a closed-loop system through microparticle filters into the air waste system of the hospital.

Whenever feasible, at least three cycles were performed at 6-week intervals.

\section{Safety strategy and workplace measurements}

A stringent occupational health safety policy was elaborated by the German pioneer group ${ }^{26}$. Briefly, three different levels of containment were proposed, namely air-tight pneumoperitoneum (balloon trocars, zero flow); laminar air flow and dilution capacity of the operating room; and remote application of cytostatics from outside the operating room. Air contamination levels were measured under clinical conditions while applying PIPAC in two patients. No leakage occurred, and no air contamination by cisplatin could be detected under real working conditions, both in surgeon and anaesthetist working positions. The tests were compliant with European Community safety law and regulations.

\section{Potential chemopotentiating combinations}

To combine the effects of PIPAC with hyperthermia, a Korean group ${ }^{34}$ developed a device to test the feasibility of hyperthermic PIPAC in five pigs. The device was coupled 
Table 1 Characteristics of preclinical studies

\begin{tabular}{|c|c|c|c|c|c|}
\hline Reference & Study type & $\begin{array}{l}\text { Primary } \\
\text { tumour }\end{array}$ & Model & Research question & Outcome/conclusions \\
\hline Jacquet et al. ${ }^{30}$ & Experimental & n.a. & In vivo rat & $\begin{array}{l}\text { Influence of increasing } \\
\text { intra-abdominal pressure on } \\
\text { tissue distribution and } \\
\text { penetration }\end{array}$ & $\begin{array}{l}\text { Increased tissue uptake in bladder, } \\
\text { diaphragm and abdominal wall }\end{array}$ \\
\hline Reymond et al. ${ }^{11}$ & Experimental & n.a. & In vivo porcine & $\begin{array}{l}\text { Development of a device to create } \\
\text { therapeutic capnoperitoneum }\end{array}$ & $\begin{array}{l}\text { Distribution in entire peritoneal } \\
\text { surface; better tissue } \\
\text { penetration under pressure }\end{array}$ \\
\hline Esquis et al. ${ }^{31}$ & $\begin{array}{l}\text { Experimental } \\
\text { comparative }\end{array}$ & CRC & In vivo rat + porcine & $\begin{array}{l}\text { Influence of increasing } \\
\text { intra-abdominal pressure on } \\
\text { penetration and antitumour } \\
\text { effectiveness }\end{array}$ & $\begin{array}{l}\text { Increased tumour accumulation } \\
\text { and antitumour effect with } \\
\text { manageable tolerance }\end{array}$ \\
\hline Facy et al. ${ }^{32}$ & $\begin{array}{l}\text { Experimental } \\
\text { comparative }\end{array}$ & CRC & In vivo porcine & $\begin{array}{l}\text { Effect of hyperthermia and high } \\
\text { pressure on blood and tissue } \\
\text { absorption of oxaliplatin }\end{array}$ & $\begin{array}{l}\text { Combination achieves highest } \\
\text { tissue concentration, no } \\
\text { increased systemic absorption }\end{array}$ \\
\hline Solass et al. ${ }^{33}$ & $\begin{array}{l}\text { Proof of concept/ } \\
\text { experimental }\end{array}$ & EC & Ex vivo human & $\begin{array}{l}\text { Combination of Dbait and } \\
\text { capnoperitoneum to evaluate } \\
\text { biodistribution and tumour } \\
\text { penetration }\end{array}$ & $\begin{array}{l}\text { Enhanced penetration; } \mathrm{CO}_{2} \text { as } \\
\text { carrier ensures drug distribution, } \\
\text { excellent intracellular uptake }\end{array}$ \\
\hline Solass et al. ${ }^{7}$ & $\begin{array}{r}\text { Technical note/ } \\
\text { experimental }\end{array}$ & n.a. & In vivo porcine & $\begin{array}{l}\text { Development of a device and } \\
\text { comparison with conventional } \\
\text { peritoneal lavage }\end{array}$ & $\begin{array}{l}\text { Distribution and penetration } \\
\text { significantly improved } \\
\text { compared with peritoneal } \\
\text { lavage }\end{array}$ \\
\hline Solass et al. ${ }^{26}$ & $\begin{array}{l}\text { Experimental/ } \\
\text { case series }\end{array}$ & n.a. & 2 patients & $\begin{array}{l}\text { Occupational health and safety } \\
\text { aspects }\end{array}$ & $\begin{array}{l}\text { Safety measures sufficient within } \\
\text { specified study conditions; no } \\
\text { occupational health hazards }\end{array}$ \\
\hline Jung et al. ${ }^{34}$ & Experimental & n.a. & In vivo porcine & $\begin{array}{l}\text { Feasibility and safety of } \\
\text { application of hyperthermic } \\
\text { PIPAC }\left(38.8-40^{\circ} \mathrm{C}\right)\end{array}$ & $\begin{array}{l}\text { Steady hyperthermic } \\
\text { capnoperitoneum achieved. } \\
\text { Feasible and safe. }\end{array}$ \\
\hline Kakchekeeva et al. ${ }^{35}$ & $\begin{array}{l}\text { Experimental } \\
\text { comparative }\end{array}$ & n.a. & In vivo porcine & $\begin{array}{l}\text { Investigation of ePIPAC for } \\
\text { enhancement of tissue uptake } \\
\text { and distribution of tracer }\end{array}$ & $\begin{array}{l}\text { Technically feasible, improved } \\
\text { tissue uptake of tracers up to } \\
\text { tenfold }\end{array}$ \\
\hline Solass et al. ${ }^{29}$ & Guidelines & n.a. & n.a. & $\begin{array}{l}\text { Development of uniform } \\
\text { terminology/grading of } \\
\text { histological tumour response }\end{array}$ & $\begin{array}{l}\text { Proposal of PRGS; needs external } \\
\text { validation }\end{array}$ \\
\hline Khosrawipour et al. ${ }^{36}$ & Experimental & n.a. & Ex vivo porcine & $\begin{array}{l}\text { Impact of irradiation on tissue } \\
\text { penetration depth of } \\
\text { doxorubicin during PIPAC }\end{array}$ & $\begin{array}{l}\text { Higher (fractional) radiation might } \\
\text { reduce penetration depth. } \\
\text { Needs further evaluation }\end{array}$ \\
\hline Khosrawipour et al. ${ }^{37}$ & Experimental & n.a. & Ex vivo porcine & $\begin{array}{l}\text { Exploration of spatial drug } \\
\text { distribution and penetration } \\
\text { pattern of doxorubicin }\end{array}$ & $\begin{array}{l}\text { Heterogeneous spatial drug } \\
\text { distribution, penetration highest } \\
\text { in centre of aerosol jet spray }\end{array}$ \\
\hline Rezniczek et al. ${ }^{38}$ & Retrospective & $\begin{array}{l}58 \mathrm{OC} \\
3 \mathrm{EC} \\
1 \mathrm{PMP} \\
1 \mathrm{GC}\end{array}$ & Ex vivo biopsies & $\begin{array}{l}\text { Analysis of gene expression } \\
\text { changes during PIPAC with } \\
\text { doxorubicin and cisplatin }\end{array}$ & $\begin{array}{l}\text { Changes in gene expression } \\
\text { patterns during repeated PIPAC } \\
\text { prognostic of overall survival }\end{array}$ \\
\hline Khosrawipour et al. ${ }^{39}$ & Experimental & n.a. & Ex vivo porcine & $\begin{array}{l}\text { Exploration of spatial drug } \\
\text { distribution pattern of } \\
\text { doxorubicin in post-mortem } \\
\text { swine }\end{array}$ & $\begin{array}{l}\text { Doxorubicin reaches all areas in } \\
\text { peritoneum; highest penetration } \\
\text { around jet spray }(350 \mu \mathrm{m})\end{array}$ \\
\hline Göhler et al. ${ }^{40}$ & Experimental & n.a. & Ex vivo analysis & $\begin{array}{l}\text { Technical description of } \mathrm{MIP}^{\circledR} \text { and } \\
\text { granulometric characterization } \\
\text { of applied aerosol }\end{array}$ & $\begin{array}{l}\text { Aerodynamics and large droplet } \\
\text { size }(>3 \mu \mathrm{m}) \text { impede } \\
\text { homogeneous drug distribution }\end{array}$ \\
\hline Khosrawipour et al. ${ }^{41}$ & Experimental & n.a. & Ex vivo porcine & $\begin{array}{l}\text { Influence of changes in pressure, } \\
\text { distance of MIP }{ }^{\circledR} \text { and } \\
\text { doxorubicin concentration on } \\
\text { penetration }\end{array}$ & $\begin{array}{l}\text { Increased with higher drug } \\
\text { concentration and closer } \mathrm{MIP}^{\circledR} \\
\text { but not with higher pressure }\end{array}$ \\
\hline
\end{tabular}

n.a., Not available; CRC, colorectal cancer; EC, endometrial cancer; Dbait, DNA repair inhibitor; PIPAC, pressurized intraperitoneal aerosol chemotherapy; ePIPAC, electrostatic precipitation as an adjunct to PIPAC; PRGS, Peritoneal Regression Grading Score; OC, ovarian cancer; PMP, pseudomyxoma peritonei; GC, gastric cancer; $M \mathrm{P}^{\circledR}$, microinjection pump (Reger Medizintechnik, Villingendorf, Germany). 
Table 2 Characteristics of clinical studies

\begin{tabular}{|c|c|c|c|c|c|c|c|c|c|c|}
\hline Reference & Study design & $\begin{array}{l}\text { No of } \\
\text { patients } \\
(\mathrm{M}: \mathrm{F})\end{array}$ & $\begin{array}{c}\text { No. of } \\
\text { PIPAC } \\
\text { procedures }\end{array}$ & $\begin{array}{l}\text { Primary } \\
\text { tumour }\end{array}$ & $\begin{array}{l}\text { Failed } \\
\text { access } \\
(\%)\end{array}$ & $\begin{array}{c}\text { Histological } \\
\text { response } \\
(\%) \ddagger\end{array}$ & $\begin{array}{l}\text { Surgery- } \\
\text { related } \\
\text { complications }\end{array}$ & $\begin{array}{l}\text { Toxicity } \\
\text { (grade } \\
\text { 1/2/3/4/5)§ }\end{array}$ & Follow-up* & Survival $^{*}$ \\
\hline $\begin{array}{l}\text { Giger-Pabst } \\
\text { et al. }{ }^{28}\end{array}$ & $\begin{array}{l}\text { Prospective } \\
\text { case series }\end{array}$ & 10 & 22 & GC & n.a. & $\begin{array}{l}\text { PP: } 100 \\
\text { ITT: } 70\end{array}$ & 9 & $\begin{array}{c}\text { n.a./n.a./ } \\
1 / 0 / 1\end{array}$ & n.a. & $>300$ days $\mathbb{I}$ \\
\hline $\begin{array}{l}\text { Blanco } \\
\text { et al. }{ }^{42}\end{array}$ & $\begin{array}{c}\text { Prospective } \\
\text { phase } 0 \\
\text { (toxicity) study }\end{array}$ & $3(2: 1)$ & 8 & $\begin{array}{c}1 \mathrm{OC}, 1 \mathrm{GC}, \\
1 \mathrm{AC}\end{array}$ & n.a. & n.a. & n.a. & n.a. & n.a. & n.a. \\
\hline $\begin{array}{l}\text { Tempfer } \\
\text { et al. }{ }^{43}\end{array}$ & Case report & $1(0: 1)$ & 3 & PMP & 0 & Yes & 0 & $3 / 3 / 0 / 0 / 0$ & 6 months & n.a. \\
\hline $\begin{array}{l}\text { Tempfer } \\
\text { et al. }{ }^{44}\end{array}$ & $\begin{array}{l}\text { Prospective } \\
\text { case series }\end{array}$ & $\begin{array}{l}21(0: 21) \\
(+8 \text { CRS })\end{array}$ & 34 & $\begin{array}{c}16 \mathrm{OC}, 4 \mathrm{PPC}, \\
1 \mathrm{FTC}\end{array}$ & 14 & PP: 75 & 12 & $12 / 0 / 3 / 2 / 0$ & 192 days & 442 days $\dagger$ \\
\hline $\begin{array}{l}\text { Solass } \\
\qquad \text { et al. }{ }^{10}\end{array}$ & $\begin{array}{l}\text { Prospective } \\
\text { case series }\end{array}$ & $3(2: 1)$ & 12 & $\begin{array}{l}1 \mathrm{GC}, 1 \mathrm{AC}, \\
1 \mathrm{OC}\end{array}$ & 0 & $\begin{array}{l}100(2 \\
\text { com- } \\
\text { plete, } 1 \\
\text { partial) }\end{array}$ & 8 & $1 / 3 / 0 / 1 / 0$ & 567 days & 288 days $\dagger$ \\
\hline $\begin{array}{l}\text { Odendahl } \\
\text { et al. }{ }^{9}\end{array}$ & $\begin{array}{l}\text { Observational, } \\
\text { retrospective }\end{array}$ & $91(51: 40)$ & 158 & $\begin{array}{l}29 \mathrm{GC}, 25 \mathrm{OC}, \\
14 \mathrm{CRC}, \\
6 \mathrm{AC}, 4 \mathrm{M}, \\
6 \mathrm{CUP}, 7 \\
\text { others }\end{array}$ & 5.5 & n.a. & $3(2)$ & $\begin{array}{c}\text { n.a./n.a./ } \\
8 / 1 / 3\end{array}$ & 12 months $\dagger$ & 13.4 months \\
\hline $\begin{array}{l}\text { Tempfer } \\
\text { et al. }{ }^{45}\end{array}$ & Phase II trial & $53(0: 53)$ & 130 & $\begin{array}{l}47 \text { OC, } 4 \text { PPC, } \\
2 \text { FTC }\end{array}$ & 17 & $\begin{array}{l}\text { PP: } 76-88 \\
\text { ITT: } 62-72\end{array}$ & $4(3)$ & $\begin{array}{c}71 / 32 / 8 / \\
0 / 0\end{array}$ & n.a. & 331 days $† \#$ \\
\hline $\begin{array}{l}\text { Tempfer } \\
\text { et al. }{ }^{46}\end{array}$ & Cohort study & $99(0: 99)$ & 252 & $\begin{array}{l}84 \mathrm{OC}, 6 \mathrm{PPC}, \\
3 \mathrm{CC}, 3 \mathrm{EC}, \\
1 \mathrm{BC}, 1 \mathrm{PMP}, \\
1 \mathrm{FTC}\end{array}$ & 17 & 76 & $5(2)$ & $\begin{array}{l}57 / 60 / 17 / \\
3 / 0\end{array}$ & 126 days & 14.1 months \\
\hline $\begin{array}{l}\text { Giger-Pabst } \\
\text { et al. }{ }^{47}\end{array}$ & Case report & $1(0: 1)$ & 8 & OC & 0 & Yes & 0 & 8/8/0/0/0 & 15 months & n.a. \\
\hline $\begin{array}{l}\text { Robella } \\
\text { et al. }{ }^{8}\end{array}$ & Retrospective & 14 & 40 & $\begin{array}{l}\text { 6GC, } 3 \mathrm{OC}, \\
2 \mathrm{CRC}, 2 \mathrm{M}, \\
1 \mathrm{PMP}\end{array}$ & 2 & n.a. & 0 & $6 / 8 / 0 / 0 / 0$ & n.a. & n.a. \\
\hline $\begin{array}{l}\text { Nadiradze } \\
\text { et al. }{ }^{48}\end{array}$ & Retrospective & $24(12: 12)$ & 60 & GC & 8 & PP : 71 & $3(5)$ & $15 / 0 / 6 / 1 / 2$ & 248 days & 15.4 months \\
\hline $\begin{array}{l}\text { Demtröder } \\
\text { et al. } 49\end{array}$ & Retrospective & $17(10: 7)$ & 42 & CRC & 13 & $71-86$ & 0 & $\begin{array}{c}\text { n.a./n.a./ } \\
4 / 0 / 0\end{array}$ & 22 months $\dagger$ & 15.7 months \\
\hline $\begin{array}{l}\text { Girshally } \\
\text { et al. }{ }^{27}\end{array}$ & Retrospective & 9 & 32 & $\begin{array}{c}6 \mathrm{CRC}+\mathrm{AC}, \\
1 \mathrm{OC}, 1 \mathrm{M}, \\
1 \mathrm{PMP}\end{array}$ & n.a. & $78-89$ & n.a. & n.a. & n.a. ${ }^{\star *}$ & n.a. ${ }^{* *}$ \\
\hline
\end{tabular}

Values in parentheses are percentages unless indicated otherwise; *values are median, except †mean. $\ddagger$ Histological response in patients who had at least one cycle of the study medication. §Common Terminology Criteria for Adverse Events grades. IIPatients with distant metastases (30 per cent) excluded. \#Intention-to-treat (ITT) analysis (mean 407 days in per-protocol (PP) analysis). **All patients had cytoreductive surgery (CRS) and hyperthermic intraperitoneal chemotherapy after neoadjuvant pressurized intraperitoneal aerosol chemotherapy (PIPAC). GC, gastric cancer; n.a., not available; OC, ovarian cancer; AC, appendiceal cancer; PMP, pseudomyxoma peritonei; PPC, primary peritoneal cancer; FTC, fallopian tube cancer; CRC, colorectal cancer; M, mesothelioma; CUP, cancer with unknown primary; CC, cervical cancer; EC, endometrial cancer, BC, breast cancer.

to heating units to achieve an effective temperature of $40^{\circ} \mathrm{C}$. The test procedures could be performed safely under these conditions, and all pigs survived for 7 days until autopsy, which showed no thermal injury to the stomach, peritoneum or jejunum.

PIPAC was combined with irradiation in an $e x$ vivo porcine model to evaluate a potential combined therapy ${ }^{36}$. Irradiation with high (fractional) radiation reduced the tissue penetration depth of doxorubicin, whereas a low (fractional) radiation dose did not.

The ability of electrostatic precipitation to enhance the physical properties of gas and pressure was evaluated ${ }^{35}$.
Electrostatic precipitation was achieved using a brush electrode for electrostatic loading of therapeutic aerosol, allowing tenfold improved tissue uptake of two tracer substances during PIPAC.

\section{Clinical experience}

\section{Feasibility and safety}

Clinical experience with PIPAC is summarized in Tables 2 and 3. Rates of failed access (unsuccessful procedures/attempted procedures) varied between 0 and 17 per cent. Surgery-related complications were observed in 
Table 3 Clinical results of studies reporting exclusively on peritoneal carcinomatosis of gynaecological, gastric and colorectal origin

$\begin{array}{lcccccc}\begin{array}{l}\text { Primary } \\ \text { cancer }\end{array} & \begin{array}{c}\text { No. of } \\ \text { studies }\end{array} & \begin{array}{c}\text { No. of } \\ \text { patients }\end{array} & \begin{array}{c}\text { No. of PIPAC } \\ \text { procedures }\end{array} & \begin{array}{c}\geq 2 \text { PIPAC } \\ \text { procedures (\%) }\end{array} & \begin{array}{c}\text { Toxicity grade } \\ 3-5(\%)^{*}\end{array} & \begin{array}{c}\text { Simultaneous } \\ \text { treatments (\%) }\end{array} \\ \text { Gynaecological } & 3^{44-46} & 184 & 416 & 38-67 & 15-28 & \text { CRS: } 44^{44} \\ \text { Gastric } & 2^{28,48} & 34 & 82 & 70-71 & 20-37 & \text { Systemic: } 33^{48} \\ \text { Colorectal } & 1^{49} & 17 & 42 & 82 & 23 & \text { Systemic: } 65\end{array}$

Case reports were not included in this analysis. ${ }^{*}$ Common Terminology Criteria for Adverse Events grades. PIPAC, pressurized intraperitoneal aerosol chemotherapy; CRS, cytoreductive surgery.

Table 4 Prospective studies on pressurized intraperitoneal chemotherapy

\begin{tabular}{|c|c|c|c|c|c|}
\hline Trial number & Country & Design & Status & Research question & Primary \\
\hline NCT02604784 & Italy & Single-arm phase II & Recruiting & $\begin{array}{l}\text { Feasibility, efficacy and safety of } \\
\text { PIPAC }\end{array}$ & $\begin{array}{c}\text { Colorectal, ovarian and gastric } \\
\text { cancer, and CUP }\end{array}$ \\
\hline NCT02735928 & Italy & Single-arm phase II & Recruiting & $\begin{array}{l}\text { Feasibility, efficacy and safety of } \\
\text { PIPAC }\end{array}$ & Ovarian cancer \\
\hline NCT02320448 & Denmark & Single-arm phase II & Active, not recruiting & Feasibility and safety of PIPAC & n.a. \\
\hline NCT01854255 & Germany & Single-arm phase II & Completed & $\begin{array}{l}\text { Feasibility, efficacy and safety of } \\
\text { PIPAC }\end{array}$ & Gastric cancer \\
\hline NCT02475772 & Germany & $\begin{array}{l}\text { Single-arm phase I } \\
\text { Dose escalation }\end{array}$ & Recruiting & $\begin{array}{l}\text { Safety and tolerability of doxorubicin } \\
\text { and cisplatin every } 4 \text { weeks using a } \\
\text { three-group, dose-escalation } \\
\text { protocol }\end{array}$ & Ovarian cancer \\
\hline
\end{tabular}

Prospective clinical trials registered at ClinicalTrials.gov on 12 December 2016. PIPAC, pressurized intraperitoneal aerosol chemotherapy; CUP, cancer with unknown primary; n.a., not available.

$0-12$ per cent; highest rates were described in a study ${ }^{44}$ that combined PIPAC with CRS, a concept that was abandoned. Postoperative adverse events were assessed by the CTCAE grading system ${ }^{12}$ in most studies. Commonly described CTCAE grade 1-2 events were abdominal pain and nausea. CTCAE grades $3-5$ were described in $0-37$ per cent; highest rates were described in a study ${ }^{44}$ that combined PIPAC with CRS and a study ${ }^{48}$ on gastric peritoneal metastases (Table 3). Two deaths were reported by Naziradze and colleagues ${ }^{48}$ in patients with gastric cancer (1 lung oedema due to ascites removal, 1 disease progression with bowel invasion), and one death from anasarca by Giger-Pabst and colleagues $^{28}$. A direct causative relationship with PIPAC was considered unlikely. Repeated PIPAC applications (at least 2) were possible in 38-82 per cent. Mean hospital stay was 3 days in the only study ${ }^{8}$ that reported on this outcome.

\section{Toxicity and systemic uptake}

Maximal peripheral venous doxorubicin concentrations after PIPAC were $4 \cdot 0-6 \cdot 2 \mathrm{ng} / \mathrm{ml}$; half-lives ranged from 86 to $468 \mathrm{~min}^{10}$. Two studies ${ }^{8,42}$ evaluated renal and hepatic toxicity, and inflammatory response. After PIPAC alone, a transient rise in serum $\gamma$-glutamyltransferase level was observed. Other liver parameters and renal tests remained within the normal range, and no cumulative toxicity was noted $^{42}$. Consistent findings were reported for PIPAC in combination with systemic chemotherapy with regard to absence of hepatorenal toxicity. Leucocytosis and an increase in C-reactive protein level were recorded after most procedures ${ }^{8}$.

\section{Efficacy}

The first human application was described in November $2011^{10}$, so long-term follow-up data were not available. The treatment response according to RECIST ${ }^{13}$ was $62-88$ per cent in the only phase II trial ${ }^{45}$. In the other studies, histopathological tumour regression was used to evaluate efficacy. An objective tumour response rate of between 62 and 100 per cent was described, but pathological assessment was inconsistent ${ }^{52,53}$ (Table 2). A novel Peritoneal Regression Grading Score was therefore developed to standardize histological assessment ${ }^{29}$ (Table 2). Median survival after PIPAC was 11-14.1 months for ovarian and gynaecological, 13.4-15.4 months for gastric and 15.7 months for colorectal peritoneal carcinomatosis.

In a recent publication ${ }^{38}$, changes in gene expression during PIPAC therapy were prognostic of overall survival, suggesting a molecular tumour response in peritoneal cancer.

\section{Quality of life}

Five publications ${ }^{8,9,45-47}$ reported on QoL after PIPAC. The German pioneer group 9 analysed QoL scores on the European Organisation for Research and Treatment of Cancer (EORTC) QLQ-30 questionnaire retrospectively in 91 patients, and described no therapy-related 
deterioration in QoL, with an absence of gastrointestinal symptoms. An independent Italian group ${ }^{8}$ confirmed these results by assessing QoL with Short Form 36 (SF-36 ${ }^{\circledR}$; Medical Outcome Trust, Health Assessment Laboratories and QualityMetric, Lincoln, Rhode Island, USA) and EORTC QLQ-30 questionnaires in 14 patients having PIPAC in combination with intravenous chemotherapy. The prospective phase II trial of Tempfer and colleagues ${ }^{45}$ demonstrated increasing EORTC QLQ-30 pain scores during PIPAC, with all patients reporting at least some form of abdominal pain. Apart from dyspnoea scores, which increased during treatment, global physical health and gastrointestinal scores improved. The same group reported improved EORTC QLQ-30 scores for global physical health, nausea/vomiting, appetite and constipation under therapy in a retrospective cohort study ${ }^{46}$.

\section{Discussion}

Preclinical data suggested improved intraperitoneal distribution and higher tissue concentrations of chemotherapy agents in PIPAC compared with HIPEC. PIPAC appeared to be safe and feasible in most patients. Clinical and histological response rates were around 70 per cent in treated patients with end-stage peritoneal disease of various origins. However, available data are limited by heterogeneity, small sample size and the absence of control groups.

PIPAC is a new technique, and no long-term results are available. Study quality was modest, and no randomized trial has yet been performed. Most studies analysed small cohorts, lacked comparative control groups and were conducted retrospectively. Study heterogeneity was high, but surgical approach and administration of PIPAC were standardized and consistent. Choice of drugs and concentrations of agents used in PIPAC were defined arbitrarily, as were further predefined conditions such as duration of administration, pressure and temperature. Of note, a dose escalation protocol has now been detailed by the pioneer centre (NCT02475772) (Table 4).

Several studies combined different treatments, mainly systemic chemotherapy and PIPAC, but even CRS was performed concomitantly in an earlier study ${ }^{44}$. Hence, observed effects cannot be attributed to PIPAC alone. On the other hand, combined treatments might increase the risk of side-effects or complications, which were still low after PIPAC even when combined with systemic chemotherapy ${ }^{8,48,49}$. This is important, as concomitant PIPAC and systemic therapy appeared to be well tolerated, also with regard to QoL during treatment ${ }^{9,45}$. A recent study ${ }^{27}$ demonstrated a potential role of PIPAC as neoadjuvant therapy before CRS/HIPEC. One of the most interesting endpoints, efficacy and tumour response, might thus be considerably biased, as it remains unclear which part of the response arises solely from PIPAC. Further, tumour response assessment was not standardized and comparisons were therefore difficult. However, this issue was addressed recently by the development of a novel grading system ${ }^{29}$.

More recent publications ${ }^{37,39-41}$ were confusing with regard to distribution and penetration of cytostatics. In clinical practice, free distribution is frequently hindered by dense adhesions or extensive peritoneal disease occluding certain areas from detailed exploration, but probably also from intraperitoneal treatment. However, accessibility of aerosolized cytostatics appeared to be superior to that achieved with intraperitoneal chemotherapy by lavage ${ }^{7}$.

Systemic treatment often represents the first-line therapy for peritoneal carcinomatosis and remains the only option in many patients owing to multifocal disease progress beyond the peritoneum. Considerable progress has been achieved with contemporary chemotherapy regimens ${ }^{54,55}$. However, outcome is worse in patients with stage IV peritoneal carcinomatosis ${ }^{56}$, underlining the need for locoregional treatments. An important drawback of the systemic approach is difficulty in the assessment of tumour response, as the reliability of imaging for diagnostic evaluation of disease progression is modest at best ${ }^{57,58}$. Therefore, peritoneal carcinomatosis remains undiagnosed in up to 50 per cent of patients ${ }^{59}$ and assessment of treatment response is feasible only either by reoperation or in the presence of a target lesion.

CRS/HIPEC is the only potentially curative option with encouraging results ${ }^{6,60,61}$. However, because of its considerable morbidity and mortality, this approach is limited to highly selected patients with favourable tumour biology and few co-morbidities. Considering the pharmacokinetic limitations $^{4,7}$ of HIPEC, PIPAC might represent an alternative for patients who are not eligible for radical treatment with curative intent, but whose disease progresses locally in the peritoneum despite systemic treatment. It should be emphasized that encouraging findings regarding feasibility, toxicity and QoL might be explained by the arbitrarily chosen low drug concentrations. Whether the good tolerance of the treatment is due to low systemic uptake alone or because of the low drug doses used in PIPAC remains to be assessed.

To date, no clear indications for PIPAC have been defined. The treatment is probably most effective in miliary peritoneal carcinomatosis, because penetration of the drug is better into small nodules than bulky deposits ${ }^{33}$. The presence of ascites might facilitate access, and Tempfer and 
colleagues ${ }^{45}$ have published data on significant reduction of ascites with repeated applications.

To optimize care, algorithms with predefined indications and contraindications to the available treatment options (systemic chemotherapy, HIPEC and PIPAC) are needed. Further studies should define standardized endpoints to allow better comparison between studies, and comparative groups are mandatory to strengthen the evidence. However, comparative studies in patients with end-stage cancer might represent a methodological and ethical challenge.

\section{Disclosure}

The authors declare no conflict of interest.

\section{References}

1 Bloemendaal AL, Verwaal VJ, van Ruth S, Boot H, Zoetmulder FA. Conventional surgery and systemic chemotherapy for peritoneal carcinomatosis of colorectal origin: a prospective study. Eur 7 Surg Oncol 2005; 31: $1145-1151$.

2 Jayne DG, Fook S, Loi C, Seow-Choen F. Peritoneal carcinomatosis from colorectal cancer. Br 7 Surg 2002; 89: $1545-1550$.

3 Sadeghi B, Arvieux C, Glehen O, Beaujard AC, Rivoire M, Baulieux J et al. Peritoneal carcinomatosis from non-gynecologic malignancies: results of the EVOCAPE 1 multicentric prospective study. Cancer 2000; 88: 358-363.

4 Dedrick RL, Flessner MF. Pharmacokinetic problems in peritoneal drug administration: tissue penetration and surface exposure. 7 Natl Cancer Inst 1997; 89: 480-487.

5 Markman M. Intraperitoneal antineoplastic drug delivery: rationale and results. Lancet Oncol 2003; 4: 277-283.

6 Glehen O, Gilly FN, Boutitie F, Bereder JM, Quenet F, Sideris $\mathrm{L}$ et al. Toward curative treatment of peritoneal carcinomatosis from nonovarian origin by cytoreductive surgery combined with perioperative intraperitoneal chemotherapy: a multi-institutional study of 1290 patients. Cancer 2010; 116: 5608-5618.

7 Solass W, Hetzel A, Nadiradze G, Sagynaliev E, Reymond MA. Description of a novel approach for intraperitoneal drug delivery and the related device. Surg Endosc 2012; 26: 1849-1855.

8 Robella M, Vaira M, De Simone M. Safety and feasibility of pressurized intraperitoneal aerosol chemotherapy (PIPAC) associated with systemic chemotherapy: an innovative approach to treat peritoneal carcinomatosis. World $\mathcal{F}$ Surg Oncol 2016; 14: 128.

9 Odendahl K, Solass W, Demtröder C, Giger-Pabst U, Zieren J, Tempfer C et al. Quality of life of patients with end-stage peritoneal metastasis treated with pressurized intraperitoneal aerosol chemotherapy (PIPAC). Eur 7 Surg Oncol 2015; 41: 1379-1385.
10 Solass W, Kerb R, Mürdter T, Giger-Pabst U, Strumberg $\mathrm{D}$, Tempfer $\mathrm{C}$ et al. Intraperitoneal chemotherapy of peritoneal carcinomatosis using pressurized aerosol as an alternative to liquid solution: first evidence for efficacy. Ann Surg Oncol 2014; 21: 553-559.

11 Reymond MA, Hu B, Garcia A, Reck T, Köckerling F, Hess $\mathrm{J}$ et al. Feasibility of therapeutic pneumoperitoneum in a large animal model using a microvaporisator. Surg Endosc 2000; 14: 51-55.

12 US Department of Health and Human Services, National Institutes of Health, National Cancer Institute. Common Terminology Criteria for Adverse Events (CTCAE), version 4.0. US Department of Health and Human Services, National Institutes of Health, National Cancer Institute: Bethesda, 2009.

13 Eisenhauer EA, Therasse P, Bogaerts J, Schwartz LH, Sargent D, Ford R et al. New response evaluation criteria in solid tumours: revised RECIST guideline (version 1.1). Eur 7 Cancer 2009; 45: 228-247.

14 Moher D, Liberati A, Tetzlaff J, Altman DG. Preferred reporting items for systematic reviews and meta-analyses: the PRISMA statement. BM7 2009; 339: b2535.

15 Schmid BC, Oehler MK. New perspectives in ovarian cancer treatment. Maturitas 2014; 77: 128-136.

16 Tempfer CB. Pressurized intraperitoneal aerosol chemotherapy as an innovative approach to treat peritoneal carcinomatosis. Med Hypotheses 2015; 85: 480-484.

17 Hübner M, Teixeira H, Boussaha T, Cachemaille M, Lehmann K, Demartines N. [PIPAC - pressurized intraperitoneal aerosol chemotherapy. A novel treatment for peritoneal carcinomatosis.] Rev Med Suisse 2015; 11: $1325-1330$.

18 Tempfer CB, Solass W, Reymond MA. Pressurized intraperitoneal chemotherapy (PIPAC) in women with gynecologic malignancies: a review. Wien Med Wochenschr 2014; 164: 519-528.

19 Sabaila A, Fauconnier A, Huchon C. [Pressurized intraperitoneal aerosol chemotherapy (PIPAC): a new way of administration in peritoneal carcinomatosis of ovarian cancer.] Gynecol Obstet Fertil 2015; 43: 66-67.

20 Glockzin G, Schlitt HJ, Piso P. Therapeutic options for peritoneal metastasis arising from colorectal cancer. World $\mathcal{F}$ Gastrointest Pharmacol Ther 2016; 7: 343-352.

21 Graversen M, Pfeiffer P, Mortensen MB. [Treatment of peritoneal carcinomatosis with pressurized intraperitoneal aerosol chemotherapy.] Ugeskr Laeger 2016; 178. pii: V11150928.

22 Haidira A, Pocard M. Nouveau mode d'administration en intrapéritonéal d'une chimiothérapie dans le traitement de la carcinose péritonéale: PIPAC (pressurized intraperitoneal aerosol chemotherapy (Master's thesis). University of Paris: Paris, 2015.

23 Reymond MA, Solass W. Pressurized Intraperitoneal Aerosol Chemotherapy - Cancer Under Pressure (1st edn). De Gruyter: Berlin, 2014. 
24 Reymond M. Therapeutic aerosolized chemotherapy for peritoneal carcinomatosis. In Eurocancer 2012. John Libbey Eurotext: Paris, 2012; 55-56.

25 Oyais A, Solass W, Zieren J, Reymond MA, Giger-Pabst U. [Occupational health aspects of pressurised intraperitoneal aerosol chemotherapy (PIPAC): confirmation of harmlessness.] Zentralbl Chir 2016; 141: 421-424.

26 Solass W, Giger-Pabst U, Zieren J, Reymond MA. Pressurized intraperitoneal aerosol chemotherapy (PIPAC): occupational health and safety aspects. Ann Surg Oncol 2013; 20: 3504-3511.

27 Girshally R, Demtröder C, Albayrak N, Zieren J, Tempfer C, Reymond MA. Pressurized intraperitoneal aerosol chemotherapy (PIPAC) as a neoadjuvant therapy before cytoreductive surgery and hyperthermic intraperitoneal chemotherapy. World 7 Surg Oncol 2016; 14: 253.

28 Giger-Pabst U, Solass W, Strumberg, D, Zieren J, Reymond M. Erste klinische Ergebnisse der 'Pressurized IntraPeritoneal Aerosol Chemotherapy' (PIPAC) bei Patienten mit Magenkarzinom und fortgeschrittener Peritonealkarzinose. Z Gastroenterol 2013; 51: K364.

29 Solass W, Sempoux C, Detlefsen S, Norman JC, Bibeau F. Peritoneal sampling and histological assessment of therapeutic response in peritoneal metastasis: proposal of the Peritoneal Regression Grading Score (PRGS). Pleura Peritoneum 2016; 1: 99-107.

30 Jacquet P, Stuart OA, Chang D, Sugarbaker PH. Effects of intra-abdominal pressure on pharmacokinetics and tissue distribution of doxorubicin after intraperitoneal administration. Anticancer Drugs 1996; 7: 596-603.

31 Esquis P, Consolo D, Magnin G, Pointaire P, Moretto P, Ynsa MD et al. High intra-abdominal pressure enhances the penetration and antitumor effect of intraperitoneal cisplatin on experimental peritoneal carcinomatosis. Ann Surg 2006; 244: 106-112.

32 Facy O, Al Samman S, Magnin G, Ghiringhelli F, Ladoire S, Chauffert B etal. High pressure enhances the effect of hyperthermia in intraperitoneal chemotherapy with oxaliplatin: an experimental study. Ann Surg 2012; 256: 1084-1088.

33 Solass W, Herbette A, Schwarz T, Hetzel A, Sun JS, Dutreix $\mathrm{M}$ et al. Therapeutic approach of human peritoneal carcinomatosis with Dbait in combination with capnoperitoneum: proof of concept. Surg Endosc 2012; 26: $847-852$.

34 Jung do H, Son SY, Oo AM, Park YS, Shin DJ, Ahn SH et al. Feasibility of hyperthermic pressurized intraperitoneal aerosol chemotherapy in a porcine model. Surg Endosc 2016; 30: 4258-4264.

35 Kakchekeeva T, Demtröder C, Herath NI, Griffiths D, Torkington J, Solass W et al. In vivo feasibility of electrostatic precipitation as an adjunct to pressurized intraperitoneal aerosol chemotherapy (ePIPAC). Ann Surg Oncol 2016; 23(Suppl 5): 592-598.

36 Khosrawipour V, Giger-Pabst U, Khosrawipour T, Pour YH, Diaz-Carballo D, Förster E et al. Effect of irradiation on tissue penetration depth of doxorubicin after pressurized intra-peritoneal aerosol chemotherapy (PIPAC) in a novel ex-vivo model. 7 Cancer 2016; 7: 910-914.

37 Khosrawipour V, Khosrawipour T, Diaz-Carballo D, Förster E, Zieren J, Giger-Pabst U. Exploring the spatial drug distribution pattern of pressurized intraperitoneal aerosol chemotherapy (PIPAC). Ann Surg Oncol 2016; 23: 1220-1224.

38 Rezniczek GA, Jüngst F, Jütte H, Tannapfel A, Hilal Z, Hefler LA et al. Dynamic changes of tumor gene expression during repeated pressurized intraperitoneal aerosol chemotherapy (PIPAC) in women with peritoneal cancer. BMC Cancer 2016; 16: 654.

39 Khosrawipour V, Khosrawipour T, Kern AJ, Osma A, Kabakci B, Diaz-Carballo D et al. Distribution pattern and penetration depth of doxorubicin after pressurized intraperitoneal aerosol chemotherapy (PIPAC) in a postmortem swine model. 7 Cancer Res Clin Oncol 2016; 142: $2275-2280$.

40 Göhler D, Khosrawipour V, Khosrawipour T, Diaz-Carballo D, Falkenstein TA, Zieren J et al. Technical description of the microinjection pump $\left(\mathrm{MIP}^{\circledR}\right)$ and granulometric characterization of the aerosol applied for pressurized intraperitoneal aerosol chemotherapy (PIPAC). Surg Endosc 2017; 31: 1778-1784.

41 Khosrawipour V, Khosrawipour T, Falkenstein TA, Diaz-Carballo D, Förster E, Osma A et al. Evaluating the effect of Micropump@ position, internal pressure and doxorubicin dosage on efficacy of pressurized intra-peritoneal aerosol chemotherapy (PIPAC) in an ex vivo model. Anticancer Res 2016; 36: 4595-4600.

42 Blanco A, Giger-Pabst U, Solass W, Zieren J, Reymond MA. Renal and hepatic toxicities after pressurized intraperitoneal aerosol chemotherapy (PIPAC). Ann Surg Oncol 2013; 20: 2311-2316.

43 Tempfer CB, Solass W, Buerkle B, Reymond MA. Pressurized intraperitoneal aerosol chemotherapy (PIPAC) with cisplatin and doxorubicin in a woman with pseudomyxoma peritonei: a case report. Gynecol Oncol Reports 2014; 10: 32-35.

44 Tempfer CB, Celik I, Solass W, Buerkle B, Pabst UG, Zieren J et al. Activity of pressurized intraperitoneal aerosol chemotherapy (PIPAC) with cisplatin and doxorubicin in women with recurrent, platinum-resistant ovarian cancer: preliminary clinical experience. Gynecol Oncol 2014; 132: $307-311$.

45 Tempfer CB, Winnekendonk G, Solass W, Horvat R, Giger-Pabst U, Zieren J et al. Pressurized intraperitoneal aerosol chemotherapy in women with recurrent ovarian cancer: a phase 2 study. Gynecol Oncol 2015; 137: 223-228.

46 Tempfer CB, Rezniczek GA, Ende P, Solass W, Reymond MA. Pressurized intraperitoneal aerosol chemotherapy with cisplatin and doxorubicin in women with peritoneal carcinomatosis: a cohort study. Anticancer Res 2015; 35: 6723-6729. 
47 Giger-Pabst U, Solass W, Buerkle B, Reymond MA, Tempfer CB. Low-dose pressurized intraperitoneal aerosol chemotherapy (PIPAC) as an alternative therapy for ovarian cancer in an octogenarian patient. Anticancer Res 2015; 35: 2309-2314.

48 Nadiradze G, Giger-Pabst U, Zieren J, Strumberg D, Solass W, Reymond MA. Pressurized intraperitoneal aerosol chemotherapy (PIPAC) with low-dose cisplatin and doxorubicin in gastric peritoneal metastasis. 7 Gastrointest Surg 2016; 20: 367-373.

49 Demtröder C, Solass W, Zieren J, Strumberg D, Giger-Pabst U, Reymond MA. Pressurized intraperitoneal aerosol chemotherapy with oxaliplatin in colorectal peritoneal metastasis. Colorectal Dis 2016; 18: 364-371.

50 da Silva RG, Sugarbaker PH. Analysis of prognostic factors in seventy patients having a complete cytoreduction plus perioperative intraperitoneal chemotherapy for carcinomatosis from colorectal cancer. 7 Am Coll Surg 2006; 203: 878-886.

51 Mazzei MA, Khader L, Cirigliano A, Cioffi Squitieri N, Guerrini S, Forzoni B etal. Accuracy of MDCT in the preoperative definition of Peritoneal Cancer Index (PCI) in patients with advanced ovarian cancer who underwent peritonectomy and hyperthermic intraperitoneal chemotherapy (HIPEC). Abdom Imaging 2013; 38: 1422-1430.

52 Glaze S, Nation J, Kobel M. Type-specific response to neoadjuvant chemotherapy: ovarian high-grade serous carcinoma versus colorectal mucinous carcinoma. 7 Obstet Gynaecol Can 2012; 34: 678-682.

53 Dworak O, Keilholz L, Hoffmann A. Pathological features of rectal cancer after preoperative radiochemotherapy. Int 7 Colorectal Dis 1997; 12: 19-23.

54 Franko J, Ibrahim Z, Gusani NJ, Holtzman MP, Bartlett DL, Zeh HJ III. Cytoreductive surgery and hyperthermic intraperitoneal chemoperfusion versus systemic chemotherapy alone for colorectal peritoneal carcinomatosis. Cancer 2010; 116: 3756-3762.

55 Elias D, Lefevre JH, Chevalier J, Brouquet A, Marchal F, Classe JM et al. Complete cytoreductive surgery plus intraperitoneal chemohyperthermia with oxaliplatin for peritoneal carcinomatosis of colorectal origin. 7 Clin Oncol 2009; 27: 681-685.

56 Franko J, Shi Q, Goldman CD, Pockaj BA, Nelson GD, Goldberg RM et al. Treatment of colorectal peritoneal carcinomatosis with systemic chemotherapy: a pooled analysis of north central cancer treatment group phase III trials N9741 and N9841. 7 Clin Oncol 2012; 30: 263-267.

57 Lambert LA. Looking up: recent advances in understanding and treating peritoneal carcinomatosis. CA Cancer 7 Clin 2015; 65: 284-298.

58 Flicek K, Ashfaq A, Johnson CD, Menias C, Bagaria S, Wasif N. Correlation of radiologic with surgical Peritoneal Cancer Index scores in patients with pseudomyxoma peritonei and peritoneal carcinomatosis: how well can we predict resectability? 7 Gastrointest Surg 2016; 20: $307-312$.

59 Elias D, Goéré D, Dumont F, Honoré C, Dartigues P, Stoclin A et al. Role of hyperthermic intraoperative peritoneal chemotherapy in the management of peritoneal metastases. Eur 7 Cancer 2014; 50: 332-340.

60 Verwaal VJ, van Ruth S, de Bree E, van Sloothen GW, van Tinteren H, Boot $\mathrm{H}$ et al. Randomized trial of cytoreduction and hyperthermic intraperitoneal chemotherapy versus systemic chemotherapy and palliative surgery in patients with peritoneal carcinomatosis of colorectal cancer. 7 Clin Oncol 2003; 21: 3737-3743.

61 Verwaal VJ. Long-term results of cytoreduction and HIPEC followed by systemic chemotherapy. Cancer 7 2009; 15: 212-215. 